\title{
Successes and Challenges in Moving On To the Future
}

\author{
MARJORIE A. CHAN1 \\ 1Dept. of Geology \& Geophysics, University of Utah, Salt
}

Lake City, UT 84112 marjorie.chan@utah.edu

In a rapidly changing world, many professional geoscience societies are worried about declining membership, and how to ensure viability for the future. As an example of a positive and successful strategy, the Geological Society of America (GSA) developed a program to encourage student diversity in race, ethnicity, gender, religion, physical and mental abilities, age, sexual orientation or marital status, national origin or ancestry, education, and class. Student membership has growth potential and thus the challenge is how to engage young people, particularly diverse populations, to help develop and foster a robust, diverse, and sustainable professional community that can lead our science.

GSA initially launched "On to the Future" (OTF) in the year of the society's 125 th anniversary, to bring and support students from underrepresented groups to their first annual GSA meeting. OTF provides partial travel funds, meeting registration, one-year of society membership, mentorship, and special orientation sessions while at the meeting. This program has now been running successfully each year since 2013, supporting a total of 647 students. The following points are key to the program success. 1) Diversity is an initiative that the society, its sections, members, donors, and foundation want to support. 2) GSA has dedicated staff, a diversity committee, and members willing to serve as mentors. 3) There is a structure for students to form a cohort that has activities to ensure professional interactions and positive experiences during the meeting. The cohort format helps the students network and build friendships that create a sense of belonging.

The end result is that diverse OTF students have been enthusiastic about this program and have expressed feelings of empowerment, and a new awareness of research and career possibilities, as well as recognizing the value of belonging to a professional society. Additionally, many societies now have ethical codes of conduct (e.g., GSA's RISE - Respectful Inclusive Scientific Events) to promote a culture and process for people to feel welcome and safe. More of these efforts to invest in diversity and community will help ensure that societies can be sustainable into the future. 